\title{
Mobile Malaria Larval Source Surveillance
}

\author{
David Ming Liu \\ Benjamin Hirsch \\ Bashir Aden \\ Ahmed Mohammad Abdalla \\ Researcher \\ Senior Researcher \\ Senior Officer \\ Senior Officer \\ Etisalat BT Innovation Center Etisalat BT Innovation Center Health Authority - Abu Dhabi Health Authority - Abu Dhabi \\ liu.ming@kustar.ac.ae \\ benjamin.hirsch@kustar.ac.ae baden@haad.ae \\ aabdalla@haad.ae
}

\begin{abstract}
Global efforts have been put in controlling malaria over past many years. Traditional Larval Source Management (LSM) which uses manual forms to keep track of the status of areas surveyed, is very inefficient in malaria control process. To address this issue, this paper describes an android mobile platform to do malaria larval source surveillance. The system has been applied by the Health Authority - Abu Dhabi (HAAD) with satisfied feedback.
\end{abstract}

Mobile Programming; Android; Malaria Control; Larval Source Management; Surveillance; GIS

\section{INTRODUCTION}

Based on the report from WHO on July 2013, there are an estimated 219 million episodes of malaria and an estimated 660,000 deaths caused by malaria in 2010. Global malaria control has been developed rapidly to prevent the malaria deaths and produced very good results. An estimated 1.1 million malaria deaths are averted over the past ten years and $58 \%$ of these lives are saved in the highest malaria rate countries like sub-Saharan Africa. Although $\mathrm{WHO}$ has declared the UAE malaria free since 2007, the potential of malaria reintroduction is high due to the large number of imported malaria from malaria endemic countries. Thus, a strong malaria surveillance systems for malaria vector is needed for planning, monitoring and evaluating of the malaria control program and hence prevention of re-introduction.

Larval Source Management is an effective way in controlling malaria for many years. The purpose of LSM is to prevent the aquatic habitats of the mosquitoes' life cycle into the mature adult stage.

Larval source management conducts inspection survey through standard forms. Traditionally inspectors have to manually fill in those forms in the fields then submit them to the concerned agencies for action. This is time consuming.

To address this issue and improve the whole process of LSM surveillance, we have built up an android mobile platform for the inspectors to enter surveillance data and synchronize it with the central server directly. The system has been applied by the Health Authority - Abu Dhabi (HAAD) with satisfied results.

The rest of the paper is organized as follows: In Section II we list out the challenges for this platform, especially in the Middle East. In Section III we describe our system architecture and show the user interfaces. In Section IV field trials of site tracking is reported in general. We conclude with current progress and future work in Section $\mathrm{V}$.

\section{CHALLENGE}

Compared to the traditional manual LSM way where surveillance data are processed manually, information and communications technology (ICT) solutions can be a big improvement in timing and saving human efforts, however it also brings some challenges.

To surveillance data, the system needs to cater for the different scenarios for different region, city and area and take down the GPS location coordinates, preferably with the geographical map display.

Synchronizing with the central server is also another challenge. The network might be very weak or sometimes totally unreachable in some remote areas, so the system must have both online and offline synchronize mode to make sure the recorded data can be sent out instantly or later when there is internet connection.

\section{SYSTEM ARCHITECTURE}

To face the challenges, we design the Mobile Malaria Larval Source Surveillance system based on the android platform.

Figure 1 describes our android mobile framework. 


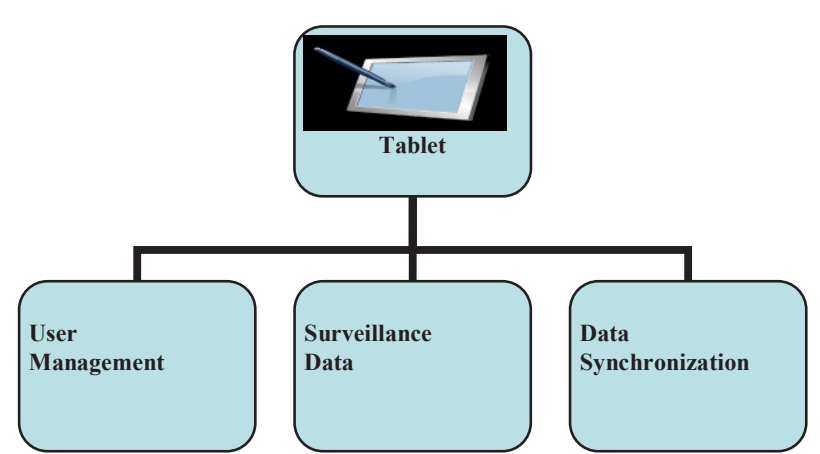

Figure 1: Android Mobile Framework

The system will have three main modules including user management, surveillance data and data synchronization. The communication between mobile and the central server is via SOAP Web Services Call. Dynamic data like the predefined region, city, area, breeding sites, type of larvae, and stage of larvae etc. will be stored in the central database and sent to mobile android application via web services.

To provide both online synchronization mode and offline synchronization mode, the survey data will be stored in the shared preferences and user can synchronize those data instantly or later based on the availability of the network.

Figure 2 shows the communication protocol between mobile and central server.

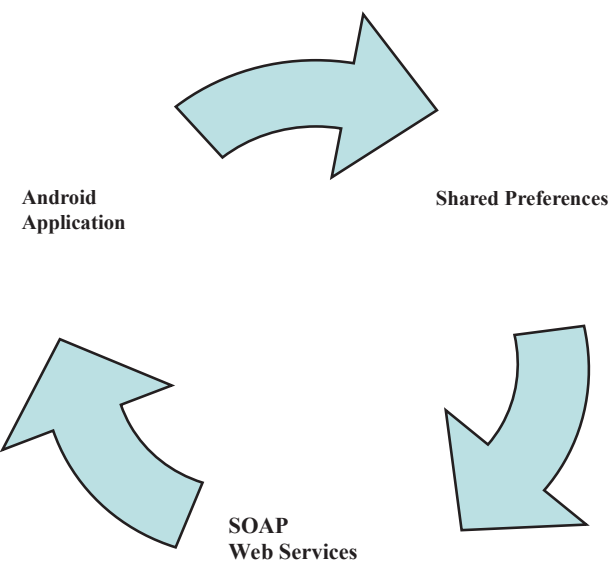

Figure 2: Android Mobile Communication Protocol

Our android mobile malaria larval source surveillance system is built up in the Samsung Galaxy Tab2 (10.1) with 3G services.

Figure 3 shows the system main interface.

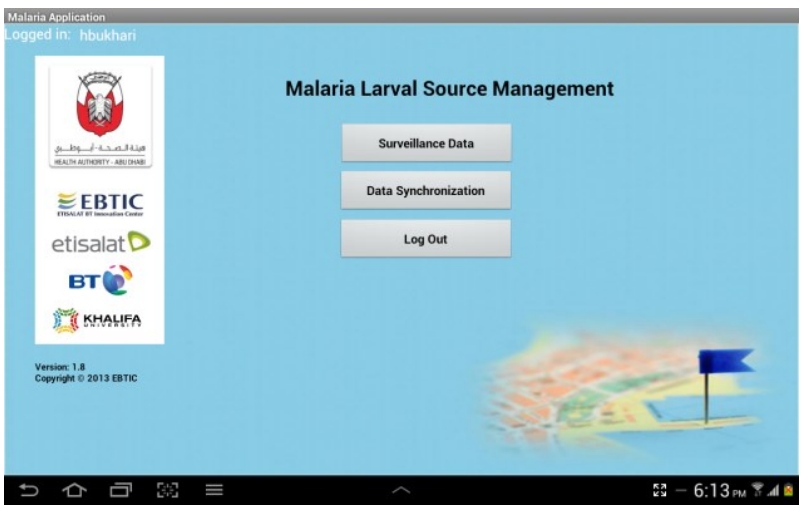

Figure 3: Main Interface

Surveillance data interface is shown in the Figure 4. The system will specify the location by Region, City, Area and locality of the mosquitoes breeding site hierarchically. We also have the GPS coordinates, accuracy and map facilities as shown in the Figure 5 for the convenience of user.

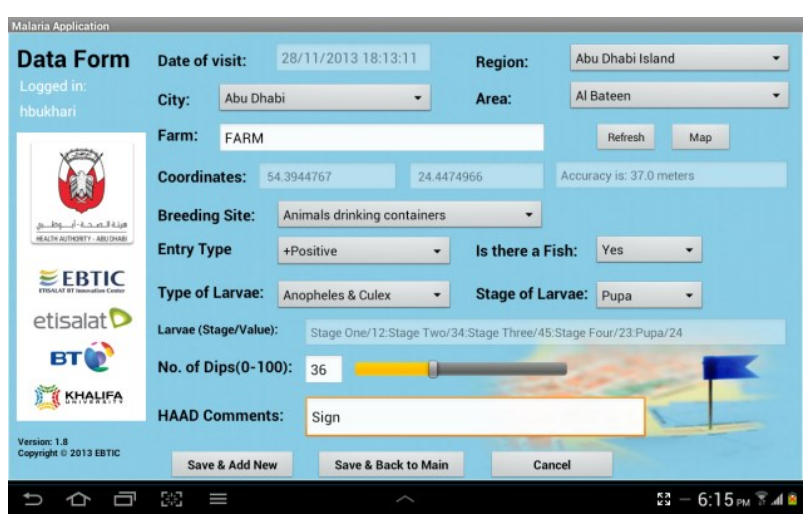

Figure 4: Surveillance Data

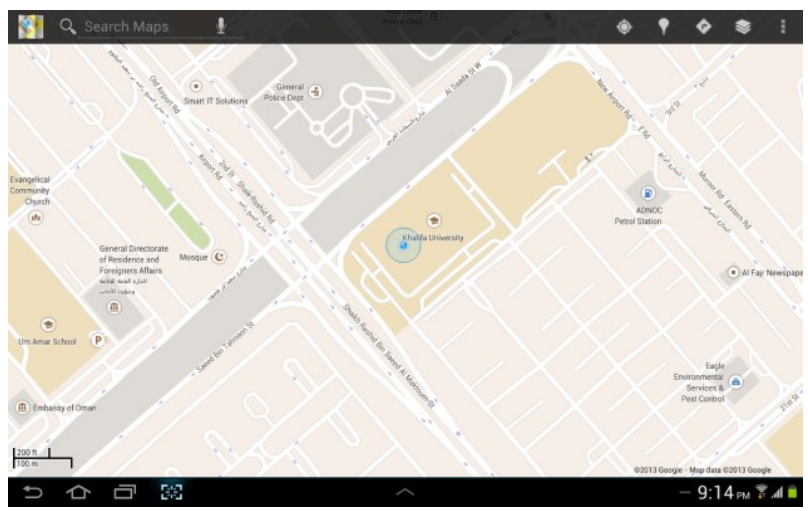

Figure 5: GIS Map

Data Synchronization is shown in the Figure 6. 


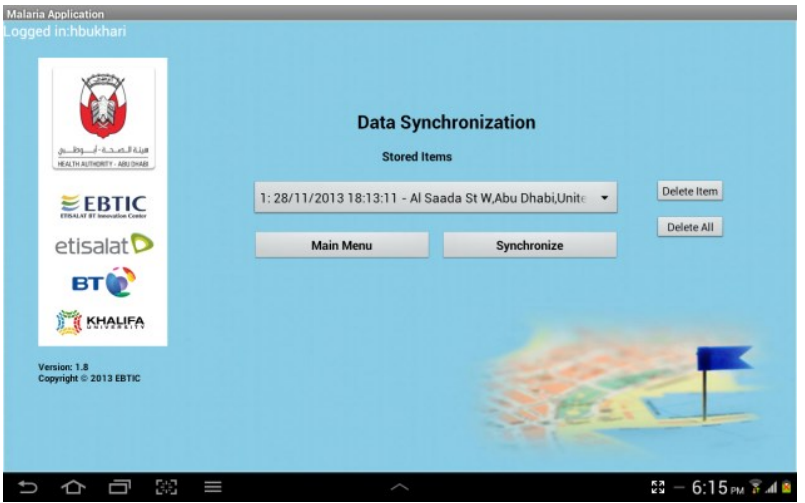

Figure 6: Data Synchronization

To provide flexible geographic location information like City/lsland and Area for the inspectors to track records, the administration module is shown in the Figure 7.

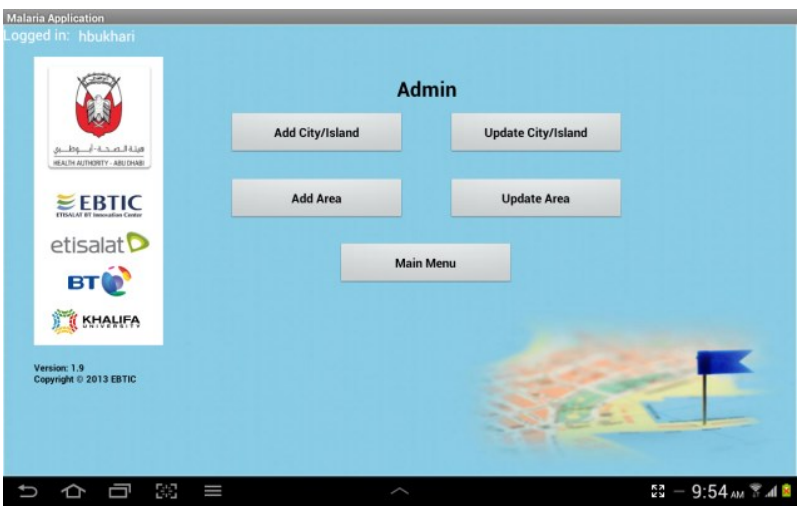

Figure 7: Administration Module

Adding and Updating City/lsland of the Region accordingly are shown in the Figure 8 and Figure 9.

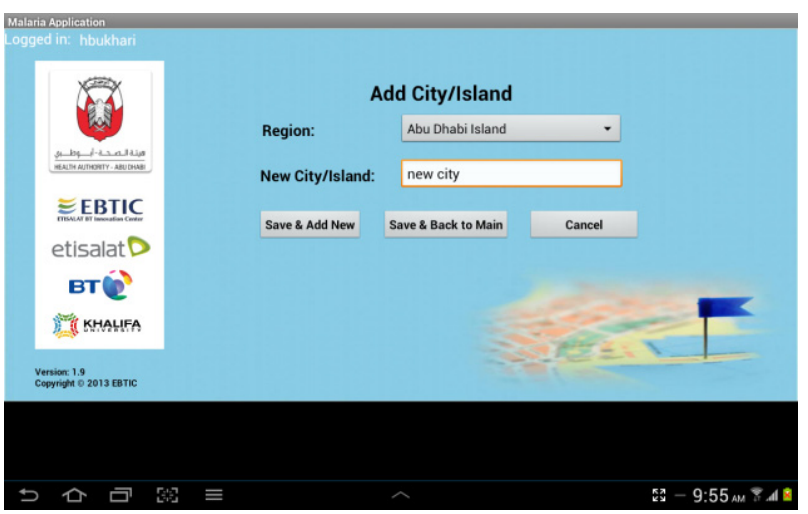

Figure 8: Add City/Island

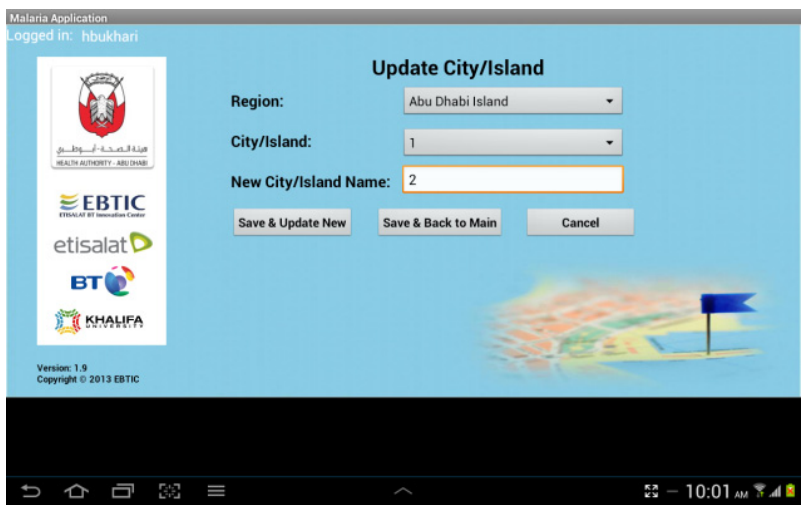

Figure 9: Update City/Island

For the Areas under the City/lsland of the Region, the adding and updating operations are shown in the Figure 10 and Figure 11.

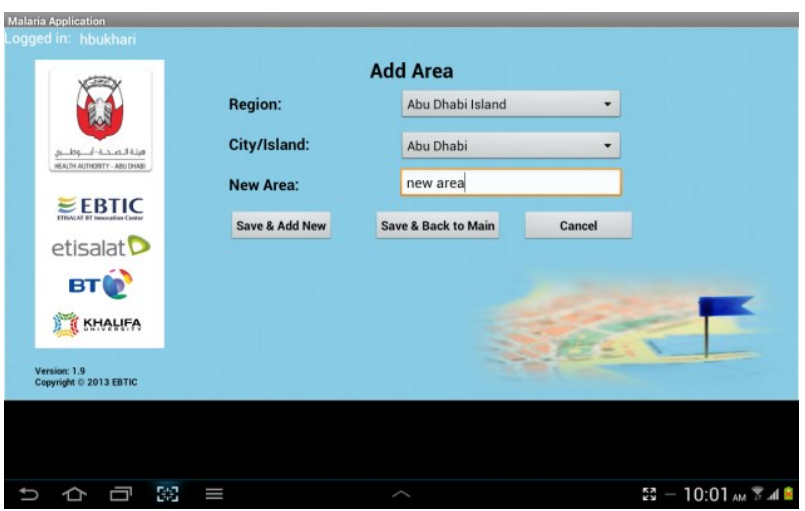

Figure 10: Add Area

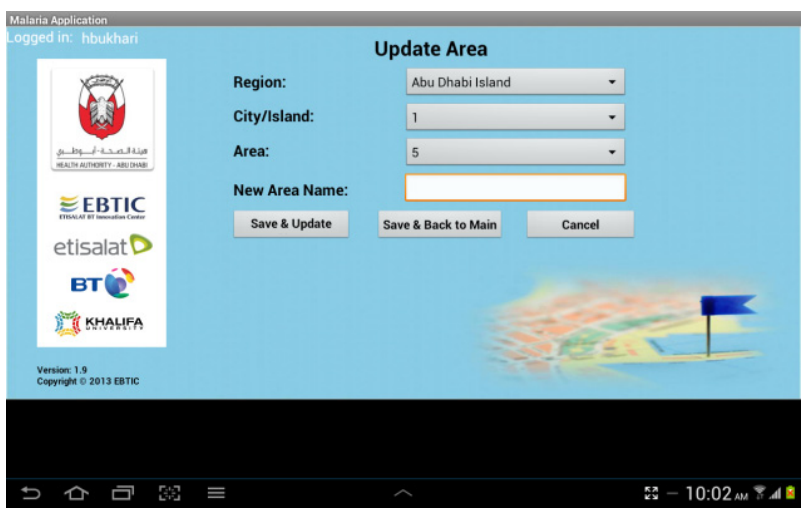

Figure 11: Update Area

With the geographic informations of Region, City/lsland and Area, the health authority officers will be able to get the whole region reports on the Malaria Larval Source situations and monitor/take action accordingly.

\section{FIELD TRAILS}

Our android mobile malaria larval source surveillance system has been applied in the Health 
Authority - Abu Dhabi (HAAD) for the larval source management purpose. Several field trials have been conducted to test and improve the system.

Figure 12 and Figure 13 show the real field trial scenarios. The inspectors will use recommended techniques to check the potential breeding sites and use our mobile malaria larval source surveillance system on the tablet to record data.

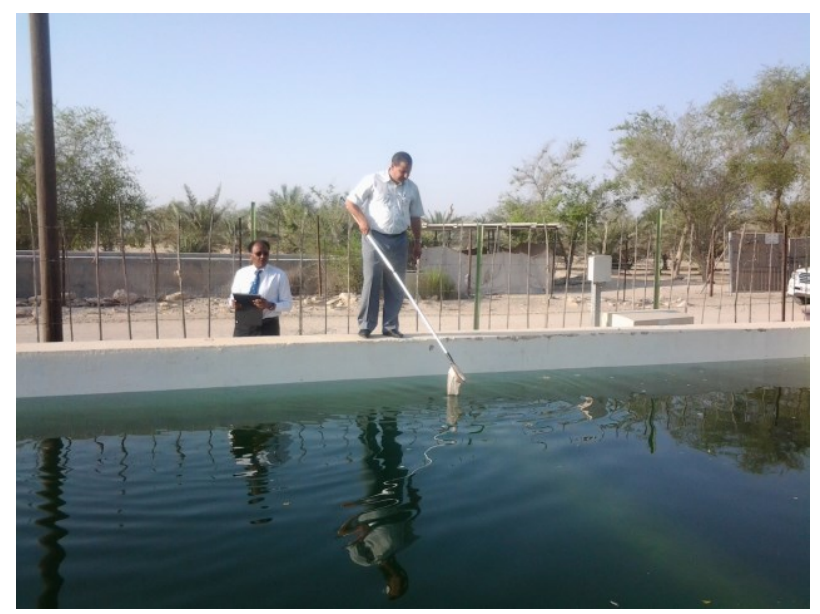

Figure 12: Field Trail

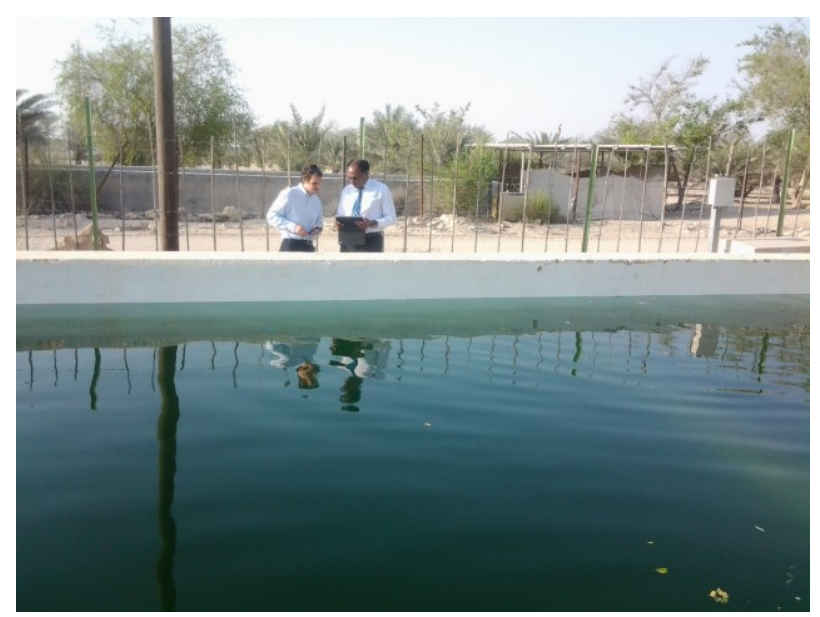

Figure 13: Surveillance Data using Tablet

We improve our system based on the field trial results to make it more user friendly and flexible. Health Authority - Abu Dhabi (HAAD) is satisfied with our system and data collection.

\section{CONCLUSION}

This android mobile malaria larval source surveillance system will change the traditional way of data entry in larval source management by applying advanced technology. It will be very efficient and user friendly for the inspectors. The whole malaria control processes will become more effective and easily to do data analysis through the central data server.
We will continue to do field trial and deploy our system into the Health Authority - Abu Dhabi (HAAD) and do the affected region analysis and prediction in the future.

\section{ACKNOWLEDGMENT}

We would like to give thanks to Mr. Hussam Mohammad Amin Al Bukhari from the Health Authority - Abu Dhabi (HAAD) for providing web services and IT support, and the Health Authority leadership for supporting the project.

\section{REFERENCES}

Larval source management - a supplementary measure for malaria vector control. An operational manual, 2013, World Health Organization.

WHO. World Malaria Report. 2012, Geneva: WHO.

WHOPES. Global insecticide use for vector-borne disease control: a 10-year assessment (20002009), 5th Ed., 2011, Geneva: WHO.

Thwing $\mathrm{J}$ et al. Mosquito larval source management for controlling malaria (Protocol). 2011, Cochrane Database of Systematic Reviews. 